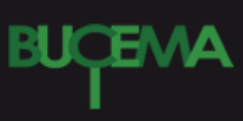

Bulletin du centre d'études médiévales d'Auxerre | BUCEMA

Hors-série $n^{\circ} 6 \mid 2013$

Autour du cloître : les chapelles Notre-Dame et les accès au chapitre

\title{
La chapelle Sainte-Marie de Cluny
}

\section{Anne Baud}

\section{(2) OpenEdition}

1 Journals

Édition électronique

URL : https://journals.openedition.org/cem/12661

DOI : $10.4000 /$ cem. 12661

ISSN : 1954-3093

Éditeur

Centre d'études médiévales Saint-Germain d'Auxerre

Référence électronique

Anne Baud, "La chapelle Sainte-Marie de Cluny », Bulletin du centre d'études médiévales d'Auxerre I BUCEMA [En ligne], Hors-série nº 6 2013, mis en ligne le 05 mars 2013, consulté le 02 mars 2023.

URL : http://journals.openedition.org/cem/12661; DOI : https://doi.org/10.4000/cem.12661

Ce document a été généré automatiquement le 2 mars 2023.

\section{(c) (i) (2)(2)}

Creative Commons - Attribution - Pas d'Utilisation Commerciale - Partage dans les Mêmes Conditions 4.0 International - CC BY-NC-SA 4.0

https://creativecommons.org/licenses/by-nc-sa/4.0/ 


\section{La chapelle Sainte-Marie de Cluny}

\section{Anne Baud}

1 La chapelle Notre-Dame, située dans l'abbaye de Cluny, fut détruite au cours du $\mathrm{XVIII}^{\mathrm{e}}$ siècle à l'occasion de la reconstruction des bâtiments monastiques. Sa situation exacte est toutefois bien connue grâce au plan anonyme et aux fouilles entreprises par K. J. Conant en 1931.

2 Si cette chapelle orientale n'est pas le fait

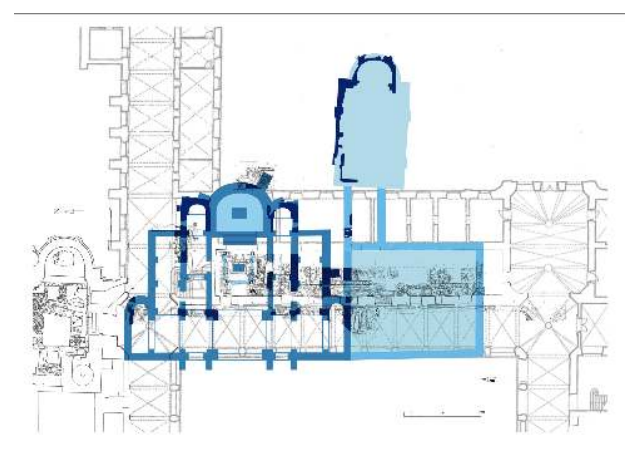
des monastères clunisiens, on note

néanmoins son développement spécifique à Cluny et dans ses prieurés au début du $\mathrm{XI}^{\mathrm{e}}$ siècle. En outre, les exemples clunisiens recensés - Charlieu, Massay, Polirone, SaintMartin-des-Champs, Saint-Germain des Prés, Souvigny, la Charité-sur-Loire, Nantua montrent que la chapelle mariale située à l'est du chevet de l'abbatiale, entre l'infirmerie et le cimetière, possède souvent une communication avec la salle capitulaire, impliquant une circulation ritualisée entre les deux lieux. Or, cette récurrence de l'organisation spatiale des bâtiments monastiques semble trouver son origine dans le développement du culte marial et le déroulement précis de la vie liturgique des moines clunisiens.

\section{Les sources iconographiques et archéologiques}


Fig. 1 : Plan anonyme (dessin de M. N. Baudrand).

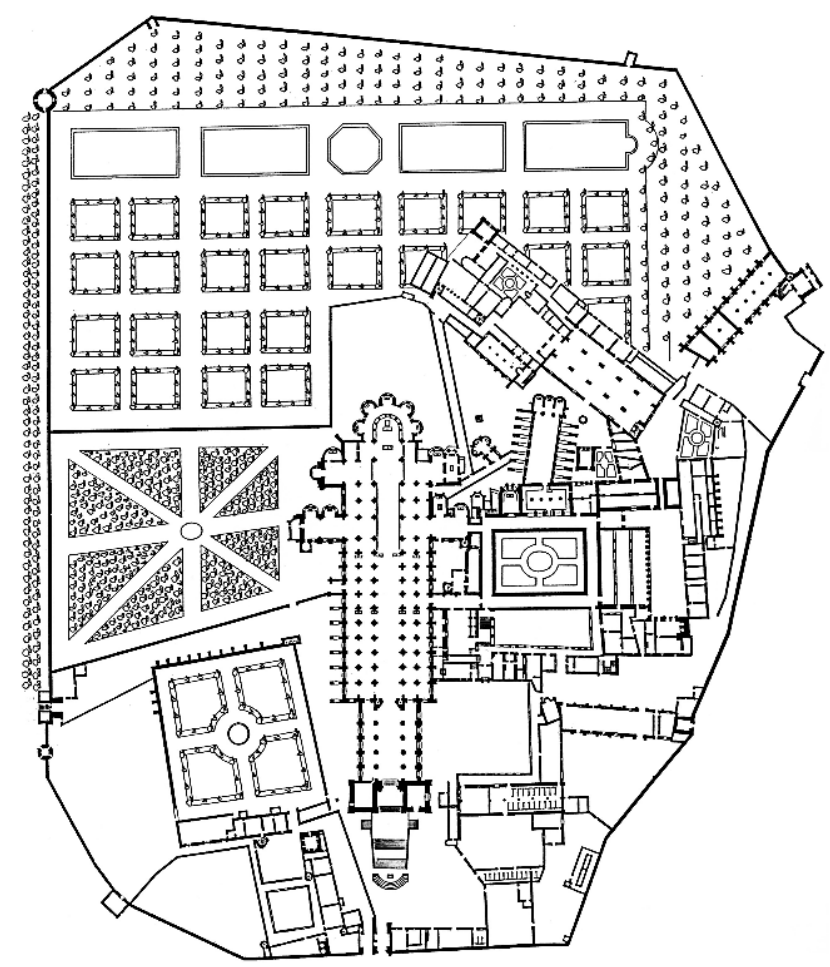

3 Le plan anonyme réalisé autour des années 1700 (fig. 1), constitue, avec le dessin de L. Prévost (vers 1670), l'unique document graphique antérieur aux grandes reconstructions de l'abbaye de Cluny qui eurent lieu au cours du XVIII ${ }^{\mathrm{e}}$. La chapelle Notre-Dame présente un plan à nef unique s'achevant à l'est par un chevet tripartite composé d'absidioles alignées. L'orientation de la chapelle diffère quelque peu de la Maior ecclesia, mais également de celle de Cluny II dont le chevet, située au nord de la salle capitulaire, fut transformé en chapelle Saint-Pierre-le-Vieux. La chapelle communique à l'ouest avec le chapitre grâce à une porte légèrement décalée vers le sud. Dès le premier tiers $\mathrm{du} \mathrm{XI}^{\mathrm{e}}$ siècle, la chapelle Sainte-Marie est mentionnée à différentes reprises dans les coutumiers de l'abbaye, notamment à l'occasion des nombreuses processions qui ponctuent le temps monastique. 
Fig. 2 : Localisation des pits.

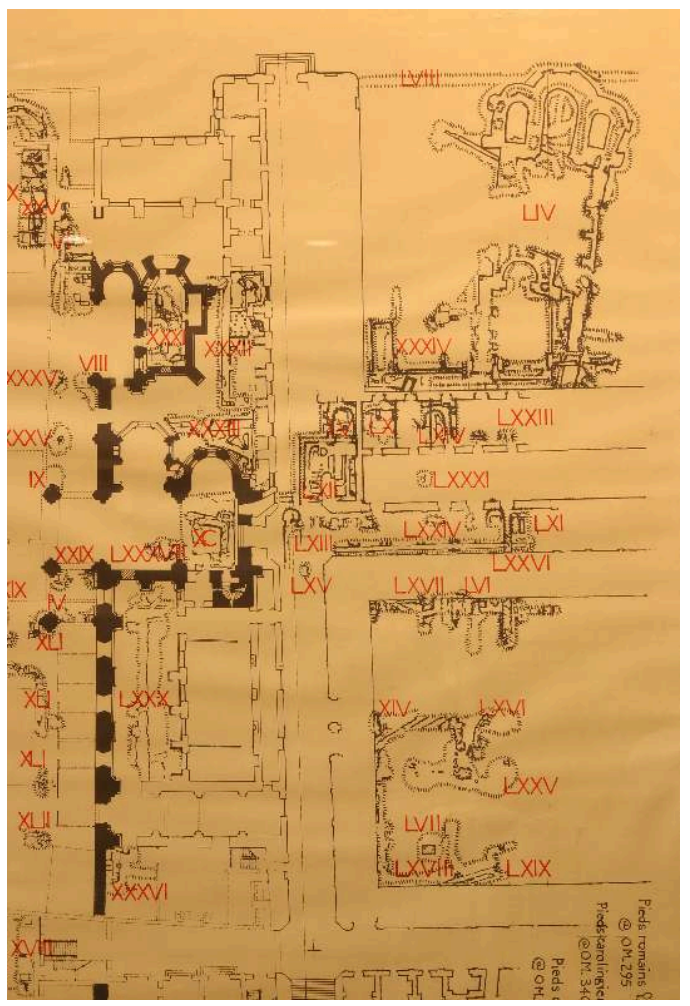

4 Les fouilles de K. J. Conant, effectuées en 1932 dans le secteur oriental de l'abbaye (pit LIV) (fig. 2), ont mis en évidence les fondations de la chapelle Notre-Dame orientée légèrement sud-est, composée d'une nef unique longue de $40 \mathrm{~m}$ environ, s'achevant par un chevet tripartite. Le plan de la chapelle correspond à celui du plan anonyme, excepté l'implantation des absidioles, qui se présentent de manière échelonnée, et non alignées. Cette chapelle remplace un édifice plus modeste, composé d'une petite nef de $10 \mathrm{~m}$ de long et doté d'une abside unique. La position de ce premier bâtiment accuse une orientation moins méridionale que le suivant. La relecture des archives de fouille par Walter Berry, montre que Conant conduisait en 1932, deux chantiers : celui de la façade de Cluny III révélant un grand nombre de lapidaires et celui de la chapelle mariale présentant a priori moins d'intérêt immédiat. Plusieurs clichés et croquis de fouilles conservés au musée Ochier à Cluny, indiquent des murs dérasés dégagés en surfaces; les nefs ont été préservées de tout sondage et les chevets successifs ont été partiellement fouillés ; les coupes précisent néanmoins la nature des maçonneries.

Les fouilles entreprises en 2011 sur le secteur de la chapelle mariale que j'ai codirigées avec C. Sapin, s'inscrivent à la suite de la campagne archéologique de 2006-2009 qui s'était déroulée dans la " galerie rouge ", soit la galerie orientale des anciens bâtiments conventuels. Celle-ci avait permis de dégager une partie du chevet de la deuxième abbatiale et la salle capitulaire dans son état gothique, tout à fait comparable, par son emplacement et ses dimensions, à celle du plan anonyme. Cette nouvelle opération menée à l'est des bâtiments conventuels, concerne les origines de l'abbaye de Cluny, avec la fouille de la demeure carolingienne, ainsi que l'origine et la fonction de la chapelle Notre-Dame. 


\section{La fonction de Sainte-Marie à la lumière des coutumiers}

6 Avant que l'archéologie n'apporte davantage d'informations sur son origine concrète et d'autres précisions notamment funéraires, la lecture des coutumiers clunisiens rédigés au cours du xie siècle, éclaire considérablement la place de la chapelle mariale au sein de la communauté clunisienne ainsi que ses diverses fonctions liturgiques. Dans la cadre de cet article, on s'arrêtera davantage sur le liber Tramitis aevi Odilonis rédigé vers 1027-1030, complété en 1033 et enfin copié autour de 1050-1060 à l'abbaye de Farfa ${ }^{1}$.

7 Dès le premier chapitre du deuxième livre du Liber tramitis, le De descriptione cluniacensis monasterii fournit divers renseignements liturgiques impliquant une hiérarchisation des bâtiments, du plus sacré au plus profane. Le claustrum, dans le sens strict du terme, est naturellement situé au cœur du monastère. Il est composé de l'église abbatiale, la salle capitulaire, l'auditorium, la camera, le dortoir, les latrines, le chauffoir, les cuisines des moines, la cuisine des laïcs, et enfin le cellier et l'aumônerie qui ferment le carré du cloître sur la galilée. Si les dimensions en longueur ou en largeur sont données pour l'ensemble des bâtiments, la hauteur n'est accordée qu'aux édifices composant le carré du cloître. L'église domine avec 43 pieds de hauteur, puis le dortoir, le réfectoire et la chapelle Sainte-Marie mesurent 23 pieds de haut formant à eux trois un niveau de toiture égal. Il ne s'agit toutefois pas d'un plan quadrangulaire puisque la chapelle mariale est concrètement située à l'extérieur du carré claustral, de l'autre côté de la salle capitulaire, elle-même surmontée par le dortoir. Il ne s'agit pas non plus de chiffres exacts ${ }^{2}$.

8 Cette description du carré claustral contestable d'un point de vue géographique, s'applique, en revanche, parfaitement à la topographie liturgique de Cluny, intimement liées aux processions monastiques sur lesquelles nous reviendrons plus loin. Le statut de Sainte-Marie, le plus souvent nommée « chapelle » dans le sens d'église secondaire et selon la terminologie admise, se perçoit à travers les différents titres qui lui sont attribués. Le terme oratorium est employé à propos des nombreuses processions, ou encore dans certains cas précis comme celui d'un frère maior ayant commis une faute légère et devant se rendre dans "l'église" ou dans "l'oratoire Sainte-Marie " ${ }^{3}$. Soulignons qu'en cette circonstance, les deux églises voisines remplissent la même fonction expiatoire. Le titre d'ecclesia est pour sa part comparable à celui de l'église abbatiale. Basilica enfin fait référence à sa fonction funéraire : lors des processions ad basilicam sanctae Mariae, les moines chantent les psaumes pour les défunts ${ }^{4}$.

9 Les fonctions de la chapelle mariale sont nombreuses et diversifiées mais inscrites au cœur de la vie monastique. Parmi les processions, la plus importante est sans conteste la procession dominicale suivie de celles des grandes fêtes : $1^{\text {er }}$ dimanche de l'Avent, Noël, la saint Sylvestre, fêtes de la Circoncision, de la Purification, de la Pentecôte et des saints Pierre et saint Paul. Selon le coutumier, ces processions monastiques conduisent la communauté, après Tierce, de l'église abbatiale à la chapelle SainteMarie, avant de rejoindre de nouveau l'église par le cloître pour la célébration de la messe conventuelle. La communauté se rend à Sainte-Marie en chantant des antiennes ou repons marials et, parvenue à l'autel de la Vierge, elle entonne le verset et une oraison en son honneur. 
10 La dévotion à la Vierge des moines clunisiens apparait, semble-t-il, dès l'abbatiat d'Odon qui appelle Marie «la Mère de miséricorde ${ }^{5}$; elle s'affirme sous Maïeul et surtout sous l'abbé Odilon connu pour ses homélies en l'honneur de la Vierge ${ }^{6}$, mais également à travers l'organisation liturgique des temps et des lieux monastiques. Marie, Vierge et mère de Dieu est donnée aux moines comme modèle : elle constitue l'exemple même de la prière contemplative. Son église au côté de l'abbatiale se justifie pleinement au sein du claustrum.

11 L'abbé Odilon honore la Vierge Marie en introduisant de nouveaux rituels : au moment $\mathrm{du} T$ Te Deum, lorsque les moines chantaient le verset « $T u$ ad liberandum suscepturus hominem non horruisti Virginis uterum " Odilon se prosternait pour adorer le verbe de Dieu s'incarnant en Marie ${ }^{7}$, geste que les moines ont conservé sous la forme d'une profonde inclination ${ }^{8}$. De même, l'abbatiat d'odilon est marqué par la solennité des nombreuses fêtes qui lui sont dédiées au cours de l'année liturgique, et le liber tramitis donne à la mère de Dieu une place particulièrement honorifique lors de la célébration des quatre fêtes anciennes: la Nativité (8 septembre), l'Annonciation (25 mars), la Purification (2 février) et l'Assomption (15 août). Le 2 février, la bénédiction de la Chandeleur et la distribution des cierges ont lieu dans la chapelle Sainte-Marie. Mais l'Assomption constitue probablement la plus grande fête consacrée à la mère de Dieu. À cette occasion, la chapelle de la Vierge qui accueille les moines lors de la messe matutinale, est parée avec autant de soins que l'abbatiale lors des fêtes de Noël et de Pâques ${ }^{9}$. Au cours de la célébration on lit l'Epistola Cogitis me, qui selon D. Iogna-Prat, exprime le triomphe de la Vierge Marie à travers la victoire des moines vierges et martyrs de Lérins, proposés comme modèle par leur sacrifice virginal ${ }^{10}$. Cet exemple permet de saisir le plus exactement possible le lien qui unit les clunisiens à la Vierge.

Située à proximité de l'infirmerie, comme l'indique le plan anonyme, la chapelle SainteMarie est l'église des malades qui y suivent les offices et assistent à la messe, en fonction de leur état physique ${ }^{11}$. Il est précisé que lorsqu'ils ne peuvent se lever seuls, ils doivent se faire aider par les familiers pour se rendre à l'office ${ }^{12}$. Plus tard, lors de la consécration de la deuxième chapelle construite sous l'abbatiat d'Hugues de Semur, celle-ci est clairement mentionnée sous le titre de "ecclesia infirmorum in monasterio Cluniacensis $»{ }^{13}$. Le rédacteur du Liber tramitis précise, à propos du moine parvenu aux dernières extrémités de sa vie terrestre, que « souvent, nous voyons dans le même jour, un frère finir dans cette lumière et passer dans le Christ, et aussi dans cette église même exhaler son esprit ${ }^{14}$. Dans la dévotion monastique, et plus particulièrement clunisienne fortement marquée par une spiritualité centrée sur le Christ ${ }^{15}$, la Vierge, par le biais de l'Incarnation, joue naturellement son rôle maternel en accueillant le fidèle défunt en son église. La chapelle Sainte-Marie, comme lieu transitoire entre la terre et le ciel, incarne concrètement cette dimension ecclésiale qui se manifeste très précisément lors du décès d'un moine : selon le Liber tramitis, le défunt doit être lavé et habillé puis la civière déposée à l'autel de la Vierge ${ }^{16}$. Après la grand'messe le corps est enseveli ${ }^{17}$. S'il n'est pas mentionné explicitement, que les moines au moment de leur agonie sont transportés sur un lit de cendre, au pied de l'autel de la chapelle, cela ne signifie pas pour autant que ce rituel est inexistant comme en témoigne l'exemple de la mort de l'abbé Odilon à Souvigny ${ }^{18}$. Le Liber tramitis (second ordo) insiste davantage sur le fait qu'à l'approche de la mort, le moine puisse se rendre dans la salle capitulaire pour le rite de confession mutuelle et d'absolution entre lui et la communauté. Puis, au moment de l'agonie, il est également précisé que les moines doivent se rendre à 
l'infirmerie au chevet du mourant ${ }^{19}$. Si l'on considère l'organisation des lieux, les circulations monastiques se déroulent nécessairement par la chapelle mariale. Au décès du moine, la communauté se réunit à Sainte-Marie pour la célébration des vêpres et des matines pour la mort.

La chapelle est également un lieu d'inhumations : les moines prient pour les défunts lorsqu'ils s'y rendent en procession, notamment au moment de l'onction des malades ${ }^{20}$. Les dernières fouilles à l'emplacement de l'oratoire Sainte-Marie, bien qu'à peine ébauchées, ont permis de mettre au jour des inhumations plus tardives que nous qualifierons de privilégiées ${ }^{21}$. Qu'en est-il pour le $\mathrm{xI}^{\mathrm{e}}$ siècle? Il est possible que, conformément aux prescriptions conciliaires, la basilica n'accueillait pas d'inhumation. Seules les nouvelles recherches permettront de préciser cet aspect essentiel de la liturgie funéraire clunisienne.

\section{La chapelle Sainte-Marie et la salle capitulaire}

On l'a vu précédemment, la salle capitulaire joue un rôle essentiel dans le rituel de la mort et la proximité des différents lieux, infirmerie, chapelle mariale et salle capitulaire est attestée par la liturgie monastique.

15 À l'instar de nombreux monastères clunisiens (Charlieu, La Charité-sur-Loire, Souvigny, Nantua...) l'oratoire Sainte-Marie communique ainsi directement avec la salle capitulaire ${ }^{22}$. Le plan anonyme met en évidence cette mitoyenneté des édifices qui remonte, selon cette configuration, au $\mathrm{XIII}^{\mathrm{e}}$ siècle ${ }^{23}$. Pour les périodes précédentes, les travaux archéologiques conduits dans l'ancienne galerie rouge ont permis de proposer certaines hypothèses sur l'existence d'une première salle capitulaire construite contre le bras sud de l'abbatiale de Cluny II, et donc implantée plus à l'ouest. Dans ce cas, il n'y aurait plus de liaison directe entre la chapelle mariale et la salle capitulaire puisque la position du mur occidental des deux chapelles successives est identique. En raison de son statut au sein du claustrum, on ne peut cependant imaginer une chapelle architecturalement dissociée du chapitre: la solution résiderait dans l'existence possible d'un couloir joignant les deux lieux (fig. 3) selon l'exemple de Saint-Martindes-Champs. 
Fig. 3 : Hypothèse de la liaison entre la chapelle Sainte-Marie et la salle capitulaire au début du $\mathrm{Xl}^{\mathrm{e}}$ siècle.

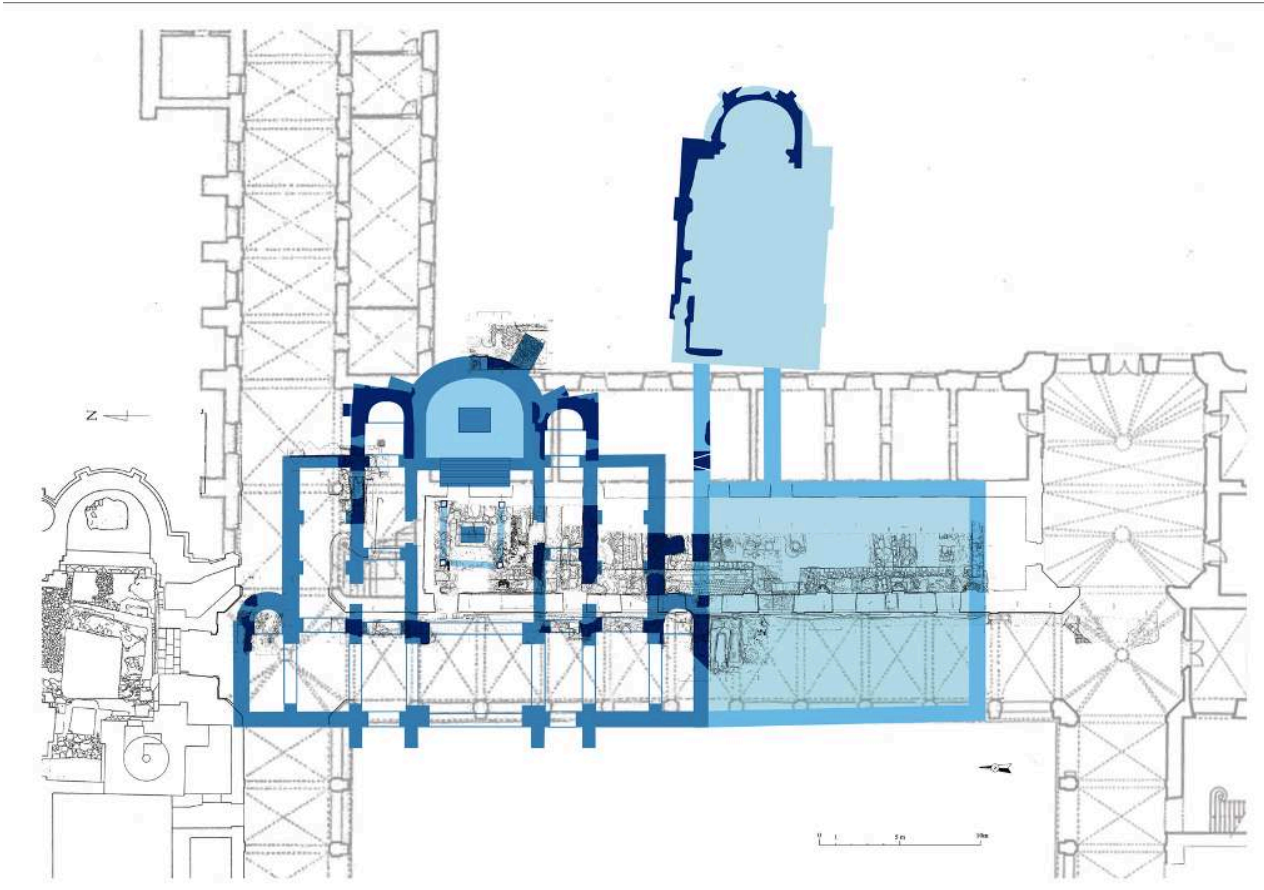

Cette liaison entre la chapelle Sainte-Marie et la salle du chapitre est confirmée par les circulations monastiques attestées à de nombreuses reprises dans les coutumiers. À l'occasion de l'onction des malades, par exemple, les moines vont directement du chapitre à la chapelle mariale tandis que les enfants restent devant l'abbatiale ${ }^{24}$. Le coutumier de Bernard précise que le moine malade logé à l'infirmerie, ne doit pas s'avancer dans la salle capitulaire, mais entrer dans l'église de la bienheureuse Marie en restant près de l'entrée tant qu'on lit le chapitre ${ }^{25}$. La liaison entre les deux bâtiments est, de fait, confirmée par la place que doit tenir le moine en cette circonstance. Par ailleurs, dans le cadre des processions monastiques $\mathrm{du} \mathrm{xI}^{\mathrm{e}}$ siècle, si l'on restitue la circulation des moines depuis l'église abbatiale jusqu'à la chapelle de la Vierge, elle ne peut s'effectuer que par la salle du chapitre.

Lorsque la troisième église abbatiale est reconstruite par Hugues de Semur à partir des années 1088, une nouvelle chapelle à la Vierge, plus grande et comprenant trois absides en son chevet, vient d'être consacrée à l'emplacement de la précédente ${ }^{26}$. Malgré le projet de reconstruction monumentale de Cluny, voulu par l'abbé Hugues, l'ancien schéma directeur des bâtiments monastiques établis sous l'abbé Odilon est conservé. La position de la salle capitulaire et de celle de la chapelle mariale reste en effet inchangée en raison de la présence de Saint-Pierre-le-Vieil, ancien sanctuaire de Cluny II : ces différents lieux du claustrum sont architecturalement liés par la liturgie processionnelle de la communauté monastique. On retrouve d'ailleurs en cela, et pour des périodes identiques, la conservation d'autres lieux aux fonctions profondément liturgiques comme l'ancienne galilée de Cluny II depuis laquelle les moines rejoignaient l'église abbatiale par le passage actuellement du même nom. Néanmoins, pour des raisons fonctionnelles, une porte située entre les deux transepts de la Maior ecclesia livre un passage direct au cimetière et à la chapelle triconque construite et consacrée au Saint 
Sépulcre. Cet accès permettait en toute logique d'arriver à la chapelle mariale sans passer par la salle capitulaire.

\section{Conclusion}

La chapelle Sainte-Marie représente probablement, avec la galilée, l'édifice monastique caractérisant le plus spécifiquement la spiritualité clunisienne, notamment à travers la place accordée à la Vierge et aux soins apportés aux défunts. En accueillant la communauté toute entière, les pénitents, les malades et les défunts, la chapelle mariale, deuxième église du claustrum après l'abbatiale, traduit également une spiritualité de type affective.

Si cette première analyse, fondée essentiellement sur les sources écrites, permet d'appréhender la signification de la chapelle mariale chez les clunisiens au début du $\mathrm{XI}^{\mathrm{e}}$ siècle, elle ne peut, cependant, apporter de réponses précises sur la date de sa construction. Dans l'état actuel de nos connaissances, le premier état pourrait remonter à la fin $d u x^{e}$ ou au tout début $d u x^{e}$ siècle. Le tout premier coutumier de la fin du $\mathrm{x}^{\mathrm{e}}$ siècle mentionne une autre église dans laquelle se rendent les moines après l'office des morts : «Post Nocturnos vero canant officium mortuorum simul. Inde veniant in alium chorum et canant matutinas de omnibus sanctis ... " ${ }^{27}$. Or alium chorum pourrait être la chapelle Sainte-Marie.

Le résultat des fouilles permettra, cependant, d'apporter de nouvelles réponses sur les origines concrètes de la chapelle consacrée à la Vierge.

\section{NOTES}

1. P. DINTER (éd.), Liber tramitis aevi odilonis abbatatis, Corpus Consuetudinum Monasticorum 10, Siegburg.

2. A. BAUD, G. ROLLIER, «Liturgie et espace monastique à Cluny à la lecture du Liber tramitis, Descriptione monasterii et données archéologiques ", in A. BAUD (dir.), Espace ecclésial et liturgie, 2006, p. 27-42.

3. LT, p. 217.20 : « Maiores autem, fratres stent in aecclesia uel in oratorio sanctae Mariae».

4. LT, p. 248 : «Quando uadum ad basilicam sanctae Marie, statim ut surrexerint, incipinat psalmos pro defunctis et postmodum prostrate pro tribulantibus absque sonitu tinnitorum ».

5. P. Cousin, «Dévotion mariale des abbés de Cluny", À Cluny, Congrès scientifique, Fêtes et cérémonies liturgiques en l'honneur des saints abbés Odon et Odilon, 9-11 juillet 1949, Dijon, 1950, p. 211, B. Gamelin, « Mater et Domina : Notre-Dame à Cluny », Cluny, Histoire, Théologie et Spiritualité, in Lettre aux amis de Solesmes, 2012-1, p. 89-95.

6. Sermons $\mathrm{n}^{\circ} 3,12,14$.

7. M. MARRIER et A. DUCHESNE (éd.), Bibliotheca Cluniacensis, Paris, 1614, réimp. Mâcon, 1915, col. 17A.

8. P. cousin, « Dévotion mariale des abbés de Cluny », À Cluny, Congrès scientifique..., p. 213. 
9. S. BOyNTON, «Les coutumes clunisiennes au temps d'Odilon », Odilon de Mercœur, l'Auvergne et Cluny, Lavoûte-Chilhac, p. 194 ; LT, p. 148.

10. D. IOGNA-PRAT, Agni immaculati, recherches sur les sources hagiographiques relatives à saint Maïeul de Cluny (954-994), Paris, 1988, p. 329 ; LT, 100.3, p. 150: «Lectiones legantur omnes ex sermone sancti Hieronimi Cogitis me o Paula ».

11. LT, p. 364 «[...] mox ut signum insonuerit,surgant et ipsi veniantque in oratorium sancte Marie ».

12. LT, p. 265 «Illi fratres qui non ualent surgere: eant famuli servientes illi et educant illos substentantes ulnis suis in ecclesia atque collocent ut melius potueint ».

13. La consécration rapportée par un manuscrit de Nantua (non retrouvé) signale que Odon, évêque d'Ostie et futur pape Urbain II procède à la dédicace de la chapelle (l'Historia reliquiarum S. Maximi apud Nantuacum - Pat. Lat. 151, col. 268). Elle aurait eu lieu en septembre 1085 ; cf. N. STRATFORD, «Les bâtiments de l'abbaye de Cluny à l'époque médiévale. État de la question », Bulletin Monumental, 1992, n. 22, p. 408.

14. LT, p. $264:$ : [...] sepe vidamus in eodem die fratrem finire ex hac luce et ad christum transire, etiam in ipsa aecclesia exalare spiritum ».

15. Th. BARBEAU, «Christologie et monachisme : le Christ au cœur de la vie des moines de Cluny », Cluny, Histoire, Théologie, Spiritualité, Lettres aux amis de Solesmes, 2012-1, p. 36-65.

16. LT, p. 274: «Signisque sonantibus ad ecclesiam primum sanctae Mariae posito feretro altare aromatizet. Responsario finito dicantur preces A porta inferi, Dominus uobiscum, Et cum spiritu tuo (et) pro illo solo singulariter oratio Deus veniae largitor, deinde altum responsorium inponentes Heu mihi domine ».

17. LT, p.275 : Post missam uero maorem sepelliendm est corpus, nisi res talis eueneit quod tandiu seruari non possit ».

18. A. MAQUET, Cluny en Auvergne, 910-1156, Thèse d'histoire médiévale, Université de Paris 1 , Panthéon-Sorbonne, M. Parisse (dir.), 2006, p. 387.

19. F. S. PAXTON, «La mort dans le coutumier du XI ${ }^{\mathrm{e}}$ siècle de Cluny ", in S. BOYTON et I. CocheliN (éd.), Du cœur de la nuit à la fin du jour: les coutumes clunisiennes au Moyen Âge, Turnhout, 2005, p. 299.

20. LT, 193, p. 269.

21. A. BAUD, C. SAPIN, Rapport intermédiaire, DRAC-SRA Bourgogne, 2011.

22. À la Charité-sur-Loire, la chapelle est dédiée à saint Laurent.

23. A. BAUD, C. SAPIN, DFS, DRAC-SRA Bourgogne, 2010.

24. LT, p. 270 : surgentes e capitulo eant in oratorium sanctae Mariae [...] Infantes cum magistris ita maneant ueluti mos est ad processionem prae regia stare. Sacerdos autem incepiat ungere infirmum ...».

25. Bernard, Ordo cluniacencis, in Vetus disciplina monastica, éd. MARQUARD HERGOTT, 1726, réimpr. P. Engelbert, 1999, p. 186 : « quamvis in capitulum non pergat, tamen intrat ecclesiam beatae Mariae prope ostium manens, quod in Capitulo legitur ».

26. Cf. note 12 .

27. CA (B et B1). 
INDEX

Index géographique : France/Cluny

Mots-clés : chapelle Sainte-Marie 